Risikoneugeborene

\section{Langzeiteffekte auf die respiratorische Gesundheit durch Frühgeburtlichkeit}

Vrijlandt EJLE et al. Respiratory Health in Adolescents Born Moderately-Late Preterm in a Community-Based Cohort. J Pediatr 2018; 203 : $429-436$

Näherungsweise kommt es jedes Jahr zu einer halben Million Frühgeburten allein in Europa und den USA, so Vrijlandt et al. Ziel der vorliegenden Studie war die Beurteilung von Langzeit-Effekten auf respiratorische und allergische Symptome, Lungenfunktion und Belastungsfähigkeit Adoleszenter nach moderater bis später Frühgeburtlichkeit in der 32.-36. Schwangerschaftswoche (SSW), entsprechend $70-85 \%$ dieser Geburten.

Die prospektive Kohortenstudie war Teil der LOLLIPOP-Studie (Longitudinal Preterm Outcome Project). Deren Schwerpunkt lag in der Beurteilung des Wachstums und der Entwicklung im Vergleich bei sehr früher Geburt (<32.SSW), moderater bis später Frühgeburt (32.-36. SSW) und bei zeitgerecht erfolgten Geburten. Die Studienkohorte war gebildet worden vor dem 3.-4. Lebensjahr aus der allgemeinen niederländischen Bevölkerung, Ausschlusskriterien waren schwere kongenitale Fehlbildungen, kongenitale Infektionen oder Syndrome. Für die vorliegende Untersuchung wurden nun Teilnehmende aus der LOLLIPOP-Studie im zwischenzeitlichen Alter von 13-14 Jahren evaluiert. Die Prävalenz von Symptomen wurde gemessen mithilfe eines Fragebogens der International Study of Asthma and Allergies in Childhood (ISAAC). Ferner wurden Lungenfunktionsuntersuchungen und Belastungstests durchgeführt. Die Rücklaufquote betrug $47 \%$ und unterschied sich nicht wesentlich bzgl. Hintergrundmerkmalen der Beteiligten.

Insgesamt nahmen 71 Jungen und Mädchen zwischen 13 und 14 Jahren an der Untersuchung teil. 37 davon waren zwi- schen der 32. und 36. SSW geboren worden, 34 hatten eine zeitgerechte Geburt erlebt. Beide Gruppen waren vergleichbar hinsichtlich Körpergröße, Körpergewicht und Ausübung von körperlichen Aktivitäten. Unterschiede bestanden neben dem Gestationsalter (34. \pm 1 SSW gegenüber 39. $\pm 0,9$ SSW) im Geburtsgewicht $(2442 \pm 539 \mathrm{~g}$ gegenüber $3693 \pm$ $393 \mathrm{~g})$. Die moderat bis spät Frühgeborenen berichteten über mehr trockenen Husten (25\% versus $3 \%, p=0,016)$ und Heuschnupfen (34\% vs. 9\%, $p=0,015$ ). Nicht vermehrt berichtet wurde über Giemen, Dyspnoe, Asthma und Ekzeme. Die Lungenfunktionsmessungen waren bei beiden Gruppen innerhalb des Normbereichs. Lediglich der exspiratorische Spitzenfluss (Gruppe der Frühgeborenen: $86 \%$ des Soll gegenüber $93 \%$ der zeitgerecht Geborenen, $p=0,05$ ) und der maximale exspiratorische Fluss bei $75 \%$ Ausatmung der forcierten Vitalkapazität $(86 \%$ vs. $96 \%, p=0,06)$ lag in der Gruppe der moderat bis spät Frühgeborenen im unteren Normbereich. Unter Belastung konnten zwischen beiden Gruppen keine Unterschiede in den Parametern festgestellt werden.

In einer Subgruppenanalyse wurden die Ergebnisse der Lungenfunktion verglichen zwischen den ehemaligen Frühgeborenen der 32.-34. SSW $(n=19)$ und der 35.-36. SSW $(n=18)$. Auch hier zeigten sich keine Unterschiede.

Einschränkend geltend gemacht wird u. a., dass die Studie im Umfang angelegt worden war auf die statistische Bewertung einzelner Lungenfunktionsparameter. So konnte z. B. ein verringertes Risiko atopischer Erkrankungen in Zusammenhang mit dem Alter bei Geburt statistisch nicht herausgearbeitet werden. Es wurden auch nur von den Teilnehmenden selbst berichtete allergische Symptome erfasst, ohne spezifische AllergieTestungen durchzuführen. Gleichzeitig beruht die Untersuchung jedoch - so Vrijlandt et al. - auf einer gut definierten repräsentativen Kohorte aus der Allgemeinbevölkerung mit präzisen Informationen bzgl. sozioökonomischer Daten und klinischer Verläufe der Neugeborenen-Periode und der frühen Kindheit.
Zusammenfassend empfohlen wird von der Arbeitsgruppe um Vrijlandt basierend auf den vorliegenden Ergebnissen, dass ein durchschnittlicher Level an körperlicher Aktivität bei 13-14-jährigen moderat bis spät Frühgeborenen angestrebt werden sollte. Dies könne als potenzieller präventiver Ansatz gelten, um das Risiko verringerter körperlicher Belastbarkeit in Wechselwirkung mit respiratorischer Gesundheit zu senken.

\section{FAZIT}

Moderate bis späte Frühgeburtlichkeit hat, so die Autorinnen und Autoren, nur geringe Auswirkungen auf die respiratorische Gesundheit in der Adoleszenz. Zwischenzeitlich 13-14 Jahre alte Betroffene berichten über wenig Symptome, diese betrafen insbesondere vermehrten (trockenen) Husten und Heuschnupfen. Die ehemaligen Frühgeborenen weisen aber nur wenig mehr Abweichungen in der Lungenfunktionsmessung auf, als zeitgerecht Geborene. In Belastungstests und körperlicher Aktivität zeigten sich keine Unterschiede.

Dr. Birgit Gappa, Kochel 